\title{
Prevalence of Hookworm infection and Strongyloidiasis in Cats and Potential Risk Factor of Human Diseases
}

\author{
Blego Sedionoto ${ }^{1,2, *}$, Witthaya Anamnart ${ }^{1}$ \\ ${ }^{1}$ Doctoral Program of Biomedical Sciences, School of Allied Health Sciences and Public Health, Walailak University, Thasala, Thailand \\ ${ }^{2}$ Department of Environmental Health, Faculty of Public Health, Mulawarman University, Samarinda- Indonesia
}

\begin{abstract}
Hookworm infection and Stronylo idiasis are public health problem in the worldwide which both of them could infective in human by penetrated on skin and they have potential risk from Gastrointestinal zoonotic helminths of pets, including cats. We investigated the prevalence soil transmitted helminths infection in human and cats used modified Formal-Ether Concentration and agar plate culture. Fecal samples of 23 cats and human from Naitung and Subua Villages (area study 1), and fecal samples of 15 cats and 17 humans from Thasala Beach villages (area study 2) were collected. Result of study in area study 1 showed prevalence of infection in human was not hookworm and strongyloidiasis but $10 \%$ humans have infected Ascaris and Tricuris, and in cats have infected by hookworm 75.2\% and S. strercoralis $8.5 \%$, toxocara $13 \%$, spirometra $13 \%$ and overall prevalence $82.5 \%$. In area study 2 showed in human has infected by Trichuris $100 \%$ and S. stercoralis $29.4 \%$ and in cats have infected by hookworm $100 \%$ and S. strercoralis $40 \%$, toxocora $20 \%$, and spirometra $20 \%$. Helminth infection found in both humans in two areas study are S. strercoralis. Hookworms were the most common helminth in cats but did not connection with infection in human, while S. strercoralis was helminth infection in cats which has potential zoonotic disease to human.
\end{abstract}

\section{Introduction}

Dogs and cats play a significant role as reservoir hosts for gastrointestinal zoonotic parasites including protozoa, trematode, cestode and nematode [1, 2, 3]. Humans can be infected via contact with a dog or cat or via contamination of infective stages in food or water [4, 5].

Worldwide, there is a significant variation in the prevalence of gastrointestinal zoonotic helminths in dogs and cats $[6,3]$. High infection rates of zoonotic parasites including hookworms, Trichuris spp., Spirometra spp., Taenia spp., Toxocara spp. and Opisthorchis spp. have been reported [7,8,6,3]. Infection of zoonotic helminths has previously been researched in Thailand.

In the central area, a high prevalence of hookworm Ancylostoma ceylanicum was reported among dogs in temple communities in Bangkok [9]. The infections of zoonotic helminths, hookworms, Trichuris spp., Toxocara spp. and Spirometra spp. were found in dogs and cats in animal refuges [10].

In the Northeastern area, a high infection rate of liver fluke, Opisthorchis viverrini (O. viverrini) in dogs and cats, was found in communities where $O$. viverrini infection in human was high [3]. In Thailand, infections of hookworms and O. viverrini are the major public health problems $[11,12,13,14,9]$.
Infections of zoonotic hookworms, A. ceylanicum and A. caninum, have been reported in many areas [13, 9]. Molecular analysis showed A. ceylanicum is prevalent in humans and dogs in the Central and the Northeastern areas of Thailand [13, 9].

Another STH, Strongyloides stercoralis, is often neglected in helminth surveys [15, 9], yet previous studies show high $S$. stercoralis infection rates in Cambodia [16]. School-aged children in the developing world are at highest risk of morbidity due to STHs and intestinal protozoan infections [17].

However, mass treatment only focuses on three major STHs (Ascaris/hookworm/Trichuris). Other nematodes like $S$. stercoralis, trematodes and protozoan infections are not addressed. In rural Southeast Asia,little is known about the zoonotic potential of IPIs in humans and animals. Therefore of domestic animals, such as cats, dogs and pigs, as contributors to human STHs and as reservoir hosts for zoonotic parasites remains unexplored and/or the data are inaccessible.

Although surveys of zoonotic gastrointestinal helminths in dogs and cats had been done in Thailand, most of the studies have focused on the Central or Northeastern region $[18,19,10,20]$. This study to investigate prevalence of zoonotic helminth infection in cats that potential risk factors to human.

* Corresponding author: blego kesling@yahoo.com 


\section{Materials and methods}

\subsection{Ethical considerations}

The study protocol was approved by the Ethics Committee of Walailak University. All participants and relevant parties were informed of the purpose of the study. Written informed consent was obtained from all individuals prior to enrolment. All infections diagnosed in humans and animals were treated at the end of the study according to the Thai treatment guidelines.

\subsection{Study design and area}

The study was carried out in 2016 at village No 4, Thasala district, Nakhon $\mathrm{Si}$ Thammarat province, Southern Thailand). The climate is tropical, with warm and hot temperatures all year round and alternating dry and wet seasons. Households from Thasala District were randomly. All household members and animals (cats) were assessed for STHs using a single diagnostic test approach on one stool sample for each human and one sample for each animal. Only animals owned by the household were included in the survey. Risk factors for infection of humans and animals were assessed based on information collected through interviews and observations.

\subsection{Field procedures and sample collection}

On the day of the first visit, informed consent was obtained from all household members and interviews were conducted with enrolled participants. Interviews with young children were conducted with the help of a parent or legal guardian.

All enrolled participants received a prelabelled stool container. Participants were asked to fill the container with feces passed the following morning. Upon collecting the first sample, a second stool container was given to participants for filling. The collected stool samples were transported within two hours following defecation to a laboratory in Medical Technology laboratory Walailak University.

Stool samples from each human and cat present at the time of the visit and belonging to the household were obtained. For each animal, approximately five grams of feces were collected from around houses, placed into a sterile plastic fecal container and chilled immediately in a box containing ice. For each human, one stool sample given on consecutive day was analysed and for each, animal, one sample was analysed.

\subsection{Laboratory procedure}

For each human stool sample, the following tests were performed: Koga Agar culture [21], formalin-ether concentration technique (FECT) [22] analysis as they arrived in the laboratory, human samples were processed as follows:

First, MFECT, Samples that were found to be negative by direct smear examination were re-examined using formol-ether concentration technique [22]. In this, about $1 \mathrm{~g}$ of the feces was collected with a stick, mixed with physiological saline and put in a screw-cap bottle containing $4 \mathrm{ml}$ of $10 \%$ formol water. The bottle was capped and mixed by shaking for about 20 seconds.

Thereafter, the feces were sieved and the suspension collected in a beaker. The suspension was poured into a tube and $3 \mathrm{ml}$ of ether was added. The tube was closed and mixed by shaking for 1 minute, after which, the stopper was removed and the setup was centrifuged immediately at 3000 r.p.m. for 1 minute. An applicator stick was used to loosen the layer of faecal debris from the side of the tube after centrifugation. The layers of ether, debris and formal water that formed were decanted off, while the sediment was mixed, transferred to a slide and covered with a cover slip. The slide was examined under the microscope using $10 \mathrm{x}$ followed by $40 \mathrm{x}$ objective to identify the hookworm eggs.

Second, a Koga Agar test was prepared by placing a piece of stool (3-5 g) on a freshly produced Agar plate. The plates were then incubated for 48 hours at $28{ }^{\circ} \mathrm{C}$. Larvae were washed from the plate into a tube, the liquid was centrifuged and the entire sediment was read at $40 \mathrm{x}$ magnification for hookworm and Strongyloides stercoralis larvae.

\section{Results}

\subsection{Humans and cats samples}

In this study, fecal samples from 15 cats at three houses Village No 4 Thasala District with 17 samples from human were collected from households Thasala Beach Village, Thasala district, Nakhon $\mathrm{Si}$ Thammarat province, Thailand.

\subsection{Prevalence in animals}

Result of study showed in two area studies such as at area study 1 hookworm $18 \quad(78.2 \%$,Strongyloidiasis 2(8.5\%), Tococara spp $3(13 \%)$, spirometra 3 (13\%) and Overal Prevalence $19(82.5 \%)$, and at area sudy 2 :hookworm infections and strongyloidiasis found in cats including hookworms 15 (100\%), Strongyloides stercoralis 6(40\%), Toxocara spp Toxocara (20\%), and Spirometra $3(20 \%)$

Table 1. Prevalence Parasite in Animals

\begin{tabular}{|l|l|l|}
\hline Parasite & Area Study 1 & Area Study 2 \\
\hline Hookworm & $78.2 \%(18 / 23)$ & $100 \%(15 / 15)$ \\
\hline $\begin{array}{l}\text { Strongyloides } \\
\text { stercoralis }\end{array}$ & $8.5 \%(2 / 23)$ & $40 \%(6 / 15)$ \\
\hline Toxocara spp & $13 \%(3 / 23)$ & $20 \%(3 / 15)$ \\
\hline Spirometra & $13 \%) 3 / 23)$ & $20 \%(3 / 15)$ \\
\hline Overall Prevalence & $82.5 \%(19 / 23)$ & - \\
\hline
\end{tabular}


Dogs and cats are important reservoir hosts of

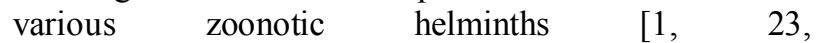
3], many of which cause serious public health problems. Result studied at Norhern Thailand reported the prevalence of zoonotic intestinal helminths as $40.1 \%$ $(79 / 197)$ in dogs and $33.9 \%(61 / 180)$ in cats, respectively.Zoonotic helminths found included hookworms, Spirometra spp., Toxocara, $O$. viverrini, Taenia spp. Strongyloides and Trichuris [24,3].

The occurrence of macroparasites was studied from 543 stray cats in four urban cities from the west (Kuala Lumpur), east (Kuantan), north (Georgetown) and south (Malacca) of Peninsular Malaysia from May 2007 to August 2010 showed up to nine species of helminths were recovered with overall high prevalences of infection of $83 \%$ in Kuantan, followed by $75.1 \%$ in Kuala Lumpur, $71.6 \%$ in Georgetown and $68 \%$ in Malacca. The helminth species comprised five nematodes, Toxocara malaysiensis, Toxocara cati, Ancylostoma braziliensis,Ancylostoma ceylanicum, Physaloptera praeputialis, two cestodes Taenia taeniaeformis, Dipylidium caninum and one trematode, Playtnosomum fastosum. [25].

Zoonotic hookworm, A. caninum, was found to have low infection rates in both dogs and cats. Similar to other areas, prevalence of $A$. caninum was lower than that of $A$. ceylanicum [21]. Although its infection rate was low, this hookworm can result in eosinophilic enteritis and chronic abdominal pain in human $[17,9]$. Other zoonotic hookworm, such as $A$. braziliensis, was not found in this area.

Among zoonotic helminth infections in dogs in the lower Northern area of Thailand, hookworms were the most prevalent helminth, and Spirometra was the second most prevalent. Our results confirmed that hookworm infection in dogs is common in Thailand [10-12]. The high prevalence of hookworm infections in dogs can contribute to the occurrence of zoonotic ancylostomiasis in human [26].

Zoonotic helminth infections in cats were different from dogs. Spirometra was the most prevalent, while hookworms were the second most prevalent helminth. High rates of Spirometra infection might be a reflection of the fact that most cats roam freely and had access to small prey as a food source. High infection rates of Spirometra spp. in cats might indicate a high infection rate of plocercoid and plerocercoid in intermediate hosts in the area. The infection of Spirometra spp. in cats and dogs can lead to a high risk of sparganosis in humans who have the habit of eating undercooked meat $[27,28]$. However, human sparganosis in Thailand is rare. In the period 1943-2010, only 53 cases had been reported [29,27].

Dogs are associated with more than 60 zoonotic parasites worldwide, many of which pose serious public health concerns [30]. Compared with some other studies in South Asian countries, the overall prevalence of IPIs in dogs in Cambodia was higher (81.9\%) than previously reported for dogs in rural India, for example [30].

This research in contrast with Combodian research that dogs in rural Cambodian villages such as Dong village are largely kept as guard dogs and allowed to roam freely, especially during the day. The dogs are also allowed inside the house and around rice and vegetable fields and ponds. At night-time, the dogs then often stay in or around the house.

Dogs, therefore, pose a serious zoonotic risk as they have the potential to transmit zoonotic parasites through their close association with household members as well as through heavy contamination of the environment, including soil, fresh produce and waterways, with parasite eggs and oocysts, in our observation sew behavior of cats almost all day and night stayed around houses and rare contact with ponds and did not stay around rice and vegetable fields.

\subsection{Prevalence in humans}

Prevalence parasite diseases in humans ain this research such as : area study $1 ; \mathrm{T}$. trichiura $10 \%(2 / 23)$ and in area study $2:$, T. Trichiura (100\%), Strongyloides strercoralis $(29.4 \%)$.

Table 2. Prevalence Parasite Infection in Human

\begin{tabular}{|l|c|c|}
\hline Parasite & Area Study 1 & Area Study 2 \\
\hline T. Trichiura & $10 \%(2 / 23)$ & $100 \%(17 / 17)$ \\
\hline $\begin{array}{l}\text { Strongyloides } \\
\text { stercoralis }\end{array}$ & - & $29.4 \%(5 / 17)$ \\
\hline Ascaris & $10 \%(2 / 23)$ & \\
\hline
\end{tabular}

The present study showed similar patterns of parasite infections in humans compared to previous surveys conducted in Cambodia [31]. The major parasite infections found in humans were hookworms $(63.3 \%)$, Entamoeba spp. (27.1\%), S. stercoralis (24.3\%), G. duodenalis (22.0\%) and Blastocystis (18.4\%) . In total, 14 different parasite species were diagnosed, including eight helminthic and six protozoan parasites. Of the 218 participants, $27(12.8 \%)$ were negative in all examinations. More than a quarter of the human participants $(64,29.4 \%)$ were infected with one parasite and a third $(72,33.0 \%)$ with two or more parasites.

Three (1.4\%) and one (0.5\%) participant(s) harboured five and six parasites, respectively, the prevalences of parasites (those with the highest infection rates) are given for the different age-groups. For hookworm, the prevalence increases from less than $50.0 \%$ in children up to the age of ten to more than $60.0 \%$ in adolescents and then remains above $60.0 \%$ in all subsequent age-groups. For $S$. stercoralis, the prevalence also increases over age, reaching its peak in age-groups 30 years and older. That research shows that the average number of helminthic co-infections increases over age, whereas the average number of protozoan coinfections is highest in children and lowest in adults older than 51 years. However, in this study, no cases of human Ascaris spp. infection were detected by microscopy. This coincides with the findings of Park and colleagues [30]. We demonstrated that in all age-groups, the average number of co-infections is about the same, 
yet helminthic co-infections accumulated over time, with a peak in 30-50 year old individuals. The trend for protozoan co-infections is reversed, with the highest number of protozoan co-infections occurring in children.

This pattern might reflect higher exposure of children. Alternatively, it could be because of higher infection intensities rather than prevalence in children, as microscopy can miss low-intensity protozoan infections [14], although this applies also for helminthic infections.

\subsection{DNA analysis}

Identify species of hookworm is N. americanus, but cats usually could be infectived by Ancylostoma caninum, condition of data this research not similar with research in Northern Thailand that it has significant zoonotic hookworms include A. ceylanicum, A. braziliensis and A. caninum $[14,28,32]$.

Molecular analysis revealed that the most prevalent hookworm (over 80\%) found in dogs and cats in the lower Northern area was A. ceylanicum. A. ceylanicum is highly prevalent in many areas in Asian countries [28, $29,34]$ and is known to produce potent infections in humans. A. ceylanicum is the second most common hookworm infection in humans that can lead to anemia $[33,27]$.

PCR and sequencing were used for detection and identification of parasites in various specimens with high sensitivity and specificity [20, 14]. In our survey, molecular analysis was applied for two significant helminths infection, hookworms and $O$. viverrini. Morphological identification of hookworm larvae or eggs to species is difficult, and molecular identification provides great results in this regard [27].

This research has deference with research in Cambodia which it showed that in humans about half of the infections (51.6\%) were Ancylostoma ceylanicum and the remaining Necator americanus infections. In dogs over $90 \%$ were $A$. ceylanicum indicating that most probably dogs are the source of infection. We hypothesize that regular deworming in communities lead to a replacement of $N$. americanus by $A$. ceylanicum. Parallel deworming of the dog population is likely to reduce the incidence in humans [14].

\subsection{Zoonotic risk factors}

Behavior of defecation cats and environmental factors also personal hygiene and sanitation have contribution became spread STHs infected from animal to human, defecation of cats observated had not potential risk infection from cats to human because cats usually closed with soil after defecated and cat anly defecated round houses and the fecal dry by solar contact then parasite was killed, deferent with studied in Nortern Thailand that Zoonotic hookworm, A. caninum, was found to have low infection rates in both dogs and cats. Similar to other areas, prevalence of $A$. caninum was lower than that of A. ceylanicum $[20,28]$. Although its infection rate was low, this hookworm can result in eosinophilic enteritis and chronic abdominal pain in human $[27,28]$.
Environmental factors have potential zoonotic determined of hookworm infection and strongylidiasis where this area researched location have poor sanitation, without wastewater drainage that made wet surrounding houses and sandy soil round houses could make easy to spread hookworm and Strongyloides stercoralis by directed penetration from cats to human, defecated cats could not spread hookworm infection and strongyloidiasis because cats behavior on defecation made larvae of soil transmitted helminth not completed to infective filariae form larvae especially hookworm and Strongyloidisis stercoralis.

\section{Conclusions}

This research given statement that cats was not equal with human hookworm infections which have not zoonotic potential also $S$. stercoralis, Ascaris lumbricoides. and T. trichiura because cats behavior on defecation made larvae of soil transmitted helminth not completed to infective filariae form larvae especially hookworm, but $S$. stercoralis became risk factors zoonotic disease by directed contact cats to human. Helminth infection found in both humans in two areas study are $S$. stercoralis. Hookworms were the most common helminth in cats but did not connection with infection in human, while $S$. strercoralis was helminth infection in cats which has potential zoonotic disease to human.

\section{References}

1. F. Fang, J. Li, T. Huang, J. Guillot, and W. Huang,. BMC Vet. Res. 11: 211, (2015)

2. A.P. Oliveira-Arbex, E.B. David, T.C. OliveiraSequeira, S. Katagiri, S.T. Coradi, and S. Guimarães, J. Helminthol. 11: 1-6, (2016)

3. Overgaauw, P.A., van Zutphen, L., Hoek, D., Yaya, F.O., Roelfsema, J., Pinelli, E., van Knapen, F. and Kortbeek, L.M. Vet. Parasitol. 163: 115-122, (2009)

4. R. Nijsse, L. Mughini-Gras, J.A. Wagenaar, F. Franssen, and H.W. Ploeger, Parasit. Vectors 8: 397, (2015)

5. V. Wiwanitkit, and W. Waenlor, Rev. Inst. Med. Trop. Sao Paulo 46:113-114, (2004)

6. D. Ng-Nguyen, S.F. Hii, V. A. Nguyen, T. Van Nguyen, D. Van Nguyen, and R.J. Traub, Parasit. Vectors 8: 40, (2015)

7. K. Ash, T. Orihel, Atlas of human parasitology, 4th edition. American Society of Clinical Pathologists, U.S.A.; (1997)

8. T. Inpankaew, F. Schär, A. Dalsgaard, V. Khieu, W. Chimnoi, C. Chhoun, D. Sok, H. Marti, S. Muth, P. Odermatt, and R. J. Traub. Emerg. Infect. Dis. 20: 976-982.(2014)

9. Q. Liu, M.W. Li, Z.D. Wang, G.H. Zhao, and X.Q. Zhu, The Lancet Infect. Dis. 15: 1226-1235, (2015) 
10. RJ. Traub, T. Inpankaew, C. Sutthikornchai, Y. Sukthana, RC. Thompson. Vet Parasitol, 155:67-73, (2008)

11. W. Rojekittikhun, K., Chaisiri, A. Mahittikorn, S. Pubampen, S. Sa-Nguankiat, T. Kusolsuk, W. Maipanich, R. Udonsom, and H. Mori, Southeast Asian J. Trop. Med. Public Health 45: 31-39, (2014)

12. A.R. Jex, Y.A. Lim, J.M. Bethony, P.J. Hotez, N.D. Young, and R.B. Gasser, Adv. Parasitol. 74:231-265, (2011)

13. P. Jongsuksuntigul, and T. Imsomboon, Acta Trop. 88: 229-232, (2003)

14. I. Phosuk, P.M. Intapan, T. Thanchomnang, O. Sanpool, P. Janwan, P. Laummaunwai, W. Anamnart, N. Morakote, and N. Maleewong, Korean J.Parasitol. 51:747-749, (2013)

15. P. Sithithaworn, R.H. Andrews, V.D. Nguyen, T. Wongsaroj, M. Sinuon, P. Odermatt, Y. Nawa, S. Liang, P.J. Brindley, and B. Sripa, Parasitol. Int. 61:10-16, (2012)

16. F. Schär, U. Trostdorf, F. Giardina, V. Khieu, S. Muth, H. Marti, et al. PLoS Negl Trop Dis,7:e2288, (2013)

17. V. Khieu, F. Schär, H. Marti, S. Sayasone, S. Duong, S. Muth, et al. PLoS Negl Trop Dis, 7:e2035, (2013)

18. B. Speich, S. Knopp, KA. Mohammed, IS. Khamis, L. Rinaldi, G. Cringoli, et al. Parasites Vectors, 3:71, (2010)

19. S. Aunpromma, P. Tangkawattana, P. Papirom, P. Kanjampa, S. Tesana, B. Sripa, and S. Tangkawattana, Parasitol. Int. 61: 60-64, (2012.)

20. T. Inpankaew, R. Traub, R.C. Thompson, and Y. Sukthana, Southeast Asian J. Trop. Med. Public Health 38: 247-255, (2007)

21. R.J. Traub, T. Inpankaew, C. Sutthikornchai, Y. Sukthana, and R.C. Thompson, Vet. Parasitol. 155: 67-73, (2008)
22. I. A. Nnanchi. O. O. Odikamnoro, A. U. Nnachi, O. C. Ani, A. C. Uhuo, C. C. Egwuatu, Nigeria International Journal of Tropical Disease \& Health 10(4). Pages: 1-10, (2015)

23. A.P. Oliveira-Arbex, E.B. David, T.C. OliveiraSequeira, S. Katagiri, S.T. Coradi, and S. Guimarães, J. Helminthol. 11: 1-6, (2016.)

24. P.A. Overgaauw, L. van Zutphen, D. Hoek, F.O. Yaya, J. Roelfsema, E. Pinelli, F. van Knapen, and L.M. Kortbeek, Vet. Parasitol. 163: 115-122, (2009)

25. Siti Nursheena Mohd Zain, Norhidayu Sahimin, Paul Pal, John W. Lewis. Veterinary Parasitology.196, Pages 469-477, (2013).

26. P. Prociv, and J. Croese, Acta Trop. 62: 23-44, (1996.)

27. Fabian Schär, Tawin Inpankaew, Rebecca J. Traub, Virak Khieuf, Anders Dalsgaard, Wissanuwat Chimnoi, Chamnan Chhoun, Daream Sok, Hanspeter Marti, Sinuon Muth, (2014)

28. P Odermatt. Parasitology International 63, 597603, (2014)

29. SK. Park, DH. Kim, YK. Deung, HJ. Kim, EJ. Yang, SJ. Lim, et al. Korean J Parasitol, 42:201-3, (2004)

30. RJ. Traub, T. Inpankaew, SA. Reid, C. Sutthikornchai, Y. Sukthana, ID. Robertson, et al. Acta Trop, 111:125-32, (2009)

31. Y.C. Hsu, and J.T. Lin, N. Engl. J. Med. 366: e20, (2012)

32. R.J. Traub, T. Inpankaew, C. Sutthikornchai, Y. Sukthana, and R.C. Thompson, Vet. Parasitol. 155: 67-73, (2008)

33. T. Inpankaew, F. Schär, A. Forrer, V. Khieu, W. Chimnoi, C. Chhoun, et al. 2012. Emerg Infect Dis 2014 [June].

34. D.D. Bowman, S.P. Montgomery, A.M. Zajac, M.L. Eberhard, and K.R. Kazacos, Hookworms of dogs and cats as agents of cutaneous larva migrans. Trends Parasitol. 26: 162-167, (2010) 